\title{
Logarithmic Chain-Exchange Kinetics of Diblock Copolymer Micelles
}

\author{
Reidar Lund, ${ }^{1, *}$ Lutz Willner, ${ }^{1}$ Jörg Stellbrink, ${ }^{1}$ Peter Lindner, ${ }^{2}$ and Dieter Richter ${ }^{1}$ \\ ${ }^{1}$ Institute for Solid State Research, Forschungszentrum Jülich, D-52425 Jülich, Germany \\ ${ }^{2}$ Institute Laue-Langevin, BP 156X, Avenue des Martyrs, F-38042 Grenoble Cedex 9, France \\ (Received 13 April 2005; published 15 February 2006)
}

\begin{abstract}
We present a study of equilibrium chain-exchange kinetics of a well-defined model system for starlike polymeric micelles. The results show that the kinetics follows a logarithmic time dependence. The same feature has been confirmed for two other micellar systems. This is in sharp contrast to theoretical predictions and hints towards strongly coupled chain dynamics within the micellar cores induced by geometrical constraints.
\end{abstract}

DOI: 10.1103/PhysRevLett.96.068302

PACS numbers: 82.70.Uv, 61.12.Ex, 61.25.Hq, 82.35.Lr

Polymeric micelles are macromolecular analogues of well-known low-molecular surfactant micelles. As a consequence of random stochastic forces, the constituting chains will continuously exchange between the micelles. From the theory of Halperin and Alexander (HA), this exchange kinetics is expected to be dominated by a simple expulsion or insertion mechanism where single chains (unimers) are required to overcome a defined potential barrier [1]. Higher order kinetics including fusion and fission is not expected to take place since these mechanisms are neither favored energetically nor entropically $[1,2]$. Experimentally, relatively few studies have been devoted to the exchange kinetics of polymeric micelles in equilibrium. This is most likely related to the associated experimental difficulties. Two principal types of methods have mainly been used: fluorescence quench spectroscopy [3-5] and temperature jump techniques [6]. However, both types of experiments generally require a significant perturbation to the equilibrium as either bulky chemical labels or strong temperature jumps are required. Nevertheless, results from fluorescence quench experiments seem to indicate a distribution of rate constants which is in contradiction to the single expulsion rate predicted in the HA model. The reason for these deviations is unknown, but speculations include the presence of bulky labels [3,5] and several kinetic mechanisms such as micellar fusion or fission [4,7] or concerted chain insertion [7]. However, since the latter mechanisms would occur in parallel, it is not immediately clear why this should lead to a large separation in the time scale.

In this Letter we demonstrate that the equilibrium exchange kinetics of starlike poly(ethylene-propylene)poly(ethylene oxide) (PEP1-PEO20, the numbers indicate the molecular weight in $\mathrm{kg} / \mathrm{mol}$ ) micelles can be described by a logarithmic time dependence. Logarithmic relaxation has been encountered in several physical situations such as in relaxation experiments on glasses [8], friction experiments [9], protein folding [10], and local dynamics of DNA [11]. In all cases the behavior is attributed to either a broadly distributed single mode or strongly coupled dy- namics. Here we will show that such logarithmic relaxation can also be found for two further micellar systems, suggesting a general phenomenon. This behavior most likely stems from strong chain correlations within the confinement of the micellar cores.

In this study we used a newly developed time resolved small angle neutron scattering (TR-SANS) technique [12]. This technique is perfectly suited for determination of exchange kinetics in equilibrium as, unlike other techniques, virtually no chemical or physical perturbations are imposed on the system. The labeling is restricted to a simple hydrogen/deuterium (H/D) substitution using fully hydrogenated (h) and fully deuterated (d) polymers with identical molar volumes and compositions. By mixing the corresponding $\mathrm{H}$ - and D-type micelles in a solvent with a scattering length corresponding to the average between the two, the kinetics can be determined. The average excess fraction of labeled chains residing inside the micelles is then simply proportional to the square root of the excess SANS intensity. The corresponding correlation function is given by $R(t)=\left\{\left[I(t)-I_{\infty}\right] /\left[I(t=0)-I_{\infty}\right]\right\}^{1 / 2}$. $I_{\infty}$ was measured from a reference sample where the polymers have been completely randomized and $I(t=0)$ from the scattering of the reservoirs at low concentrations.

This technique is applied to resolve the equilibrium exchange kinetics of a well-defined PEP1-PEO20 micellar system. This system consists of a very asymmetric PEP1-PEO20 block copolymer in water $/ N, N-$ dimethylformamide (DMF) solvent mixtures which are selective for PEO. The system has the advantage that PEP, which is the core forming polymer, has a very low glass transition temperature $\left(T_{g}=-56^{\circ} \mathrm{C}\right)$, and consequently any ambiguity about glassy dynamics in the micellar core can be excluded. The two polymers, h-PEP1-hPEO20 and d-PEP1-d-PEO20, have almost identical molar volumes with the same block composition and low polydispersities. Previous SANS investigations [13] have shown that the micelles formed are starlike, consisting of a small compact PEP core surrounded by a large extended PEO corona exhibiting strong excluded volume effects. 
TABLE I. Molecular weight characteristics of the block copolymers. $M$, molar mass; $N$, number of repeat units.

\begin{tabular}{lcccccc}
\hline \hline Polymer & \multicolumn{3}{c}{ PEP } & \multicolumn{4}{c}{ PEO } \\
\hline & $M_{n}$ & $M_{w} / M_{n}$ & $N$ & $M_{n}$ & $M_{w} / M_{n}$ & $N$ \\
\hline h-PEP1-h-PEO20 & 1100 & 1.06 & 16 & 21900 & 1.04 & 497 \\
d-PEP1-d-PEO20 & 1400 & 1.06 & 17 & 23900 & 1.04 & 497 \\
\hline \hline
\end{tabular}

Important characteristics are given in Table I and Table II. By variation of the DMF content, the interfacial tension, $\gamma$, between PEP and the solvent can be effectively tuned and used as a key control parameter for aggregation [13] and exchange kinetics [14]. Despite the fact that the PEP core is far above its glass transition, the micelles appear to be frozen in pure water where $\gamma=46 \mathrm{mN} \mathrm{m}^{-1}$. However, upon reducing the interfacial tension to $\gamma=$ $21.8 \mathrm{mN} \mathrm{m}^{-1}$ by adding $25 \mathrm{~mol} \% \mathrm{DMF}$, the kinetics occurs on a time scale $(\mathrm{min} / \mathrm{h})$ that can be easily followed by TR-SANS.

Halperin and Alexander predict that the relaxation kinetics for polymeric micelles should be described by a single exponential, namely, $R(t)=\exp (-k t)$, where the expulsion rate constant is given by

$$
k=\frac{1}{\tau_{0}} \exp \left(-E_{a} / k_{b} T\right) f\left(N_{A}, N_{B}\right)
$$

where $\tau_{0}$ is a characteristic time, $f\left(N_{A}, N_{B}\right)=$ $N_{B}^{-2 / 25} N_{A}^{-9 / 5}$ is a prefactor depending on the number of repeat units of the soluble $\left(N_{A}\right)$ or insoluble block $\left(N_{B}\right)$. The activation energy, $E_{a}$, is given by

$$
E_{a}=N_{B}^{2 / 3} l_{B}^{2} \gamma,
$$

where $l_{B}$ is the monomer length of the insoluble $B$ block.

This prediction can be compared with the experimental results for the PEP1-PEO20 micelles in $25 \mathrm{~mol} \% \mathrm{DMF}$. The results first show that the kinetics is concentration independent in the range of $\phi=0.25 \%-2 \%$ polymer volume fraction. This demonstrates that the kinetics is indeed unimolecular in nature and not diffusion controlled; i.e., the kinetics is solely determined by the expulsion rate given above. In Fig. 1 the corresponding relaxation functions, $R(t)$, at different temperatures at $\phi=1 \%$ are depicted in a semilogarithmic representation.

TABLE II. Micellar characteristics of PEP1-PEO20 in $25 \mathrm{~mol} \% \mathrm{DMF} /$ water mixture. $R_{c}$ and $R_{m}$ are the core and overall micellar radius, respectively. $P$ is the aggregation number. The micellar core is compact and free of solvent. All data at $20^{\circ} \mathrm{C}$.

\begin{tabular}{cccc}
\hline \hline System & $R_{c} / \AA$ & $R_{m} / \AA$ & $P$ \\
\hline h-PEP1-h-PEO20/DMF/water & $31 \pm 1$ & $226 \pm 2$ & $53 \pm 2$ \\
d-PEP1-d-PEO20/DMF/water & $31 \pm 1$ & $236 \pm 3$ & $57 \pm 3$ \\
\hline \hline
\end{tabular}

As clearly shown in Fig. 1, the data disagree strongly with a single exponential (straight solid line) but rather seem to exhibit a distribution of relaxation times. Such broadening may arise from a distribution of molecular weights. For the present case the polydispersity of the important core forming PEP block is low, $M_{w} / M_{n}=$ 1.06. Assuming a Poisson distribution of chain lengths around $\left\langle N_{B}\right\rangle=16$, the corresponding relaxation behavior can be estimated using

$$
R(t)=\int_{1}^{\infty} P\left(N_{B}\right) \exp (-k t) d N_{B},
$$

where $k$ is given by Eqs. (1) and (2). The Poisson distribution takes the form

$$
P\left(N_{B}\right)=\frac{\left(\left\langle N_{B}\right\rangle-1\right)^{N_{B}-1} \exp \left[-\left(\left\langle N_{B}\right\rangle-1\right)\right]}{\Gamma\left(N_{B}\right)},
$$

where $\Gamma\left(N_{B}\right)$ is the Gamma function. The results of this analysis are illustrated by the dotted lines in Fig. 1, which represent simulated curves over the whole temperature range where both $l_{B}$ and $\gamma$ are known parameters and only $\tau_{0}$ has been adjusted. As seen the data cannot be described using this scenario-the experimentally observed decay is much slower, whereas the temperature dependence is significantly stronger. Assuming the unlikely scenario that the chains do not collapse during the expulsion process but rather assume their complete linear lateral dimension, i.e., $E_{a} \sim N_{B}$, yields broader decay but cannot describe the data. The data may be fitted only if the parameters in Eqs. (1) and (2) are allowed to assume unphysical values, i.e., using a general Gaussian distribution of activation energies or a log-normal distribution of rates, give $\left\langle E_{a}\right\rangle \approx 165 \mathrm{~kJ} / \mathrm{mol}$ and $\tau_{0} \approx 1 \times 10^{-28} \mathrm{~s}$, both

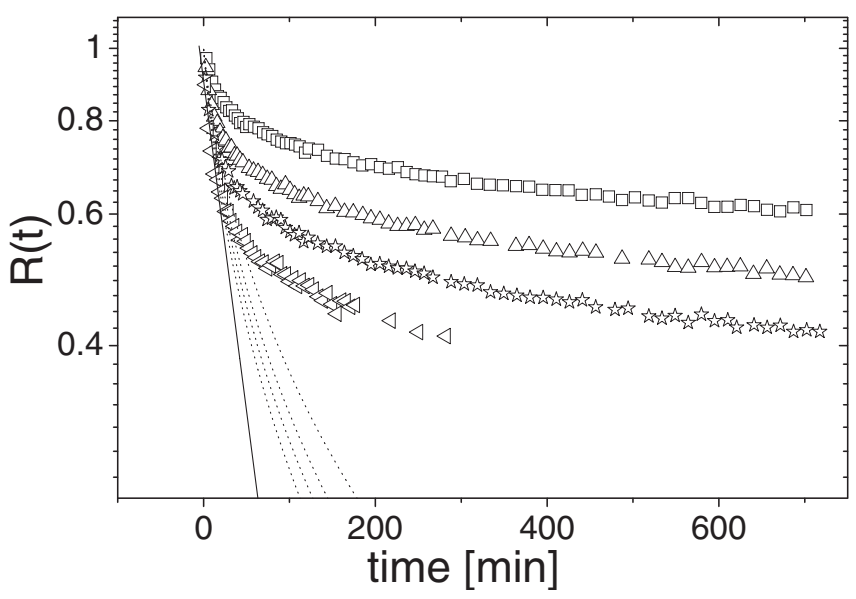

FIG. 1. Relaxation kinetics of the PEP1-PEO20 micelles in $25 \mathrm{~mol} \% \mathrm{DMF} /$ water mixture system at $\phi=1 \%$ and different temperatures in a semilogarithmical presentation. From top to bottom: 47, 55, 60, and $65^{\circ} \mathrm{C}$. The solid line displays the theoretically expected single exponential decay. The dotted lines indicate the expected theoretical decay taking into account the polydispersity of the core blocks. 
of which are clearly unreasonable. This is a strong indication that the data cannot be explained by a broad distribution of parallel independent modes. The results also seem to indicate that $\tau_{0}$ has a significant temperature dependence that cannot be accounted for by viewing the process as a simple hindered single chain diffusion such as in the HA activated jump process.

However, the observed temperature dependence could arise from a cooperativity between the chains in the micellar core. Indeed, plotting the data on a logarithmic time scale, we observe near straight lines demonstrating a clear logarithmic time dependence. This is shown in Fig. 2 and implies an extremely broad distribution without any natural characteristic time.

In terms of the expulsion rate probabilities, a logarithmic time dependence implies a distribution approximately on the form $g(k) \sim 1 / k$ for finite $k$ and $t$. Including the cutoff rate constants, $k_{\min }$, and $k_{\max }$ to take into account the finite size of the system gives the following nondiverging expression [11]:

$$
R(t)=\frac{1}{\ln \left(k_{\max } / k_{\min }\right)}\left[E_{1}\left(k_{\min } t\right)-E_{1}\left(k_{\max } t\right)\right]
$$

where $E_{1}(z)=\int_{z}^{\infty} \exp (-t) / t d t$ is the exponential integral function. The result of the fitting is given in Fig. 2.

Despite the lack of information at very short times, we can estimate a maximal initial time, defined by $\tau_{\min }=$ $1 / k_{\min }$, to be in the order of 5-0.9 min, decreasing with $T$. Assuming that $\tau_{\min }$ corresponds to the elementary expulsion rate in the HA theory in Eq. (1), we obtain $\tau_{0}$ of $\approx 1 \times 10^{-6} \mathrm{~s}$, which is in the right order of the Rouse time for the core chains. Times longer than the Rouse time may then be a signature of correlations inside the core which retard the diffusion out of the core. $\tau_{\max }=1 / k_{\max }$ cannot be determined since no deviations from the logarithmic decay are visible even at long times (up to $12-13 \mathrm{~h}$ ).

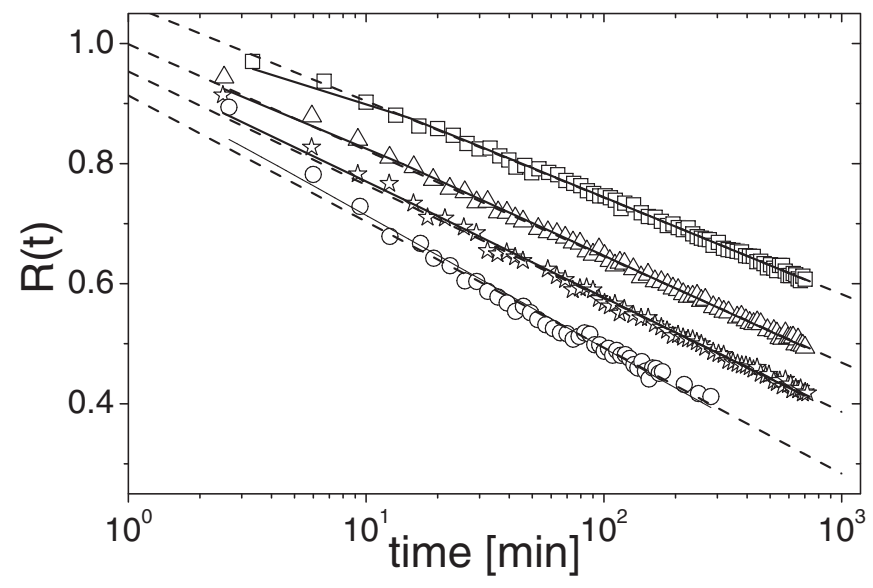

FIG. 2. Logarithmic chain-exchange kinetics. Same data as in Fig. 1 on a logarithmic time scale. The dotted lines are fits to the pure logarithmic decay and the solid lines display fits to Eq. (5).
In order to investigate the generality of the peculiar logarithmic relaxation function, the same experiments were carried out on a different block copolymer system. This system is a symmetric poly(styrene)-poly(butadiene) (PS10-PB10) block copolymer in n-hexadecane and DMF, which are selective for PB and PS, respectively. Previous studies have shown that the micelles formed are compact structures with constant density profiles of the corona [14]. However, while the micellar core of PS10-PB10/DMF system is compact, the core of the PS10-PB10/hexadecane system contains approximately $35 \%$ solvent. The results from the exchange kinetics of these micelles, which are inverted analogues to each other, are given in Fig. 3.

As observed the two inverted analogues exhibit the same near logarithmic time decay although their micellar characteristics are completely different from the PEP1PEO20/DMF/water system. As a precautionary note it should be mentioned that while $\mathrm{PB} 10$ has a very low $\mathrm{Tg}$ $\left(\approx-95^{\circ} \mathrm{C}\right.$ ), pure PS10 has a $\mathrm{Tg}$ of $\approx 100{ }^{\circ} \mathrm{C}$. However, in this case, the PS core is strongly swollen (35\% solvent) such that $\mathrm{Tg}$ is drastically moved towards lower temperature. In fact measurements showed that PS10 containing only less than $8 \%$ solvent gave a reduction of $\approx 50{ }^{\circ} \mathrm{C}$. Nevertheless, we cannot $a$ priori rule out the possibility that glassy dynamics could influence the core dynamics of the PS10-PB10/n-hexadecane system. However, in any case the results still demonstrate the same logarithmic relaxation as in the other cases.

The question now is what may cause such a broad logarithmic relaxation. As clearly demonstrated in Fig. 1, one broadly distributed mode arising from polydispersity effects cannot explain the results alone. Additional factors, such as statistical or systematic variances of the size of the micelles themselves or isotope effects, could, although weaker, contribute to a further broadening of the mode. However, as demonstrated by SANS [13,14], the individu-

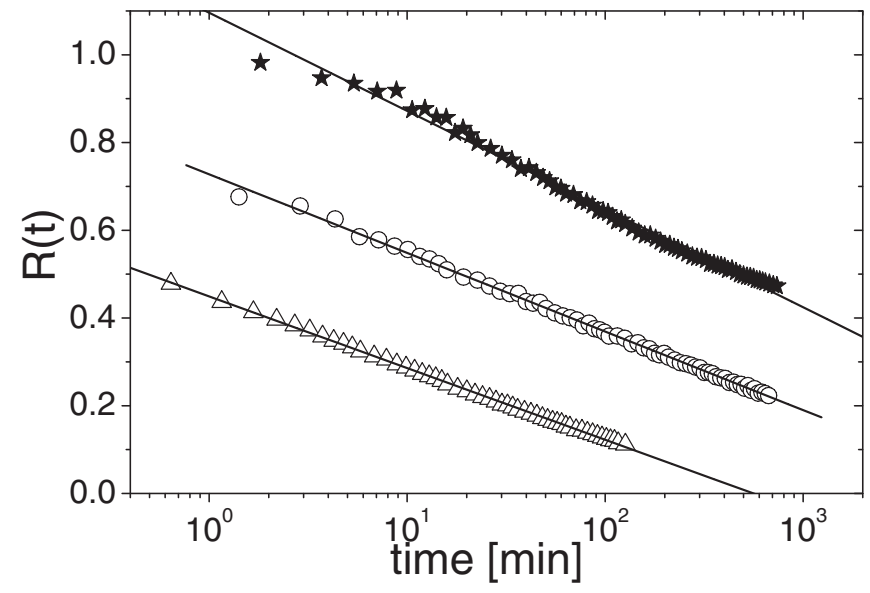

FIG. 3. Relaxation kinetics on a logarithmic time scale. From top to bottom: PS10-PB10/DMF at $20^{\circ} \mathrm{C}$ and PS10-PB10/nhexadecane at 20 and $30^{\circ} \mathrm{C}$, respectively. The solid lines display the best fits as obtained by linear regression. 
ally labeled micelles were found to have the same properties within $10 \%$ (see, e.g., Table II). We also note that any sign of broad distribution of micellar sizes or intermicellar clustering could not be identified as the SANS scattering patterns were very well defined. Also, no change in shape was observed during the kinetic measurements, demonstrating that the structure is invariant during the exchange process. Concerning isotope effects, this can be excluded, as the interfacial tensions of water to h-PEP and d-PEP, respectively, are identical [13].

Summarizing the arguments, we find that no parallel independent relaxation pathways arising from polydispersity and/or different mechanisms that can explain the logarithmic behavior. This is also demonstrated in the failure to describe the data with regular distribution functions. Additionally, the data in Fig. 1 demonstrate a temperature dependence that is much stronger than expected from the HA theory. Since the logarithmic time dependence is found in all cases, independent of system, the results suggest that the peculiar kinetics is caused by an inherent property of polymeric micelles.

A broad distribution of relaxation rates may not be caused only by parallel processes originating from a heterogeneous ensemble. In fact, as pointed out by Palmer et al. [15] heterogeneous behavior can be a consequence of "hierarchically constrained dynamics" where the relaxation occurs in individual steps in a series rather than in parallel. Later, such correlations have indeed been shown to give rise to logarithmic relaxation [16]. For polymer micelles, similar correlations may be at play in the internal core dynamics. Strong interchain correlations may be induced by the geometrical packing restrictions which confines the chains to regions of the order of 3-8 $\mathrm{nm}$. An important effect of the confinement is that long range density fluctuations are not possible. Because of the incompressibility and topological interactions between the chains, this may lead to a higher degree of cooperativity and coupling between the chains [17]. Thus rearrangement processes involving transitions between chain conformations and configurations are subject to strong dynamical constraints and are hence very slow. Such effects have indeed been seen in a recent dynamic Monte Carlo simulation study of confined chains in spherical geometry, which indicates that these structural rearrangements are slow and broadly distributed in time [18]. It is natural to assume that the probability to leave the core is only significant provided that the chain has a reasonable compact structure near the interface. Accordingly, the effective time scale of self-diffusion processes such as the chain expulsion out of the core will be broad and depend on the configuration and conformation of the chain. Similar heterogeneous behavior have been found in self-diffusion experiments of block copolymer melts with spherical geometry which show extremely broad distributions of selfdiffusion coefficients below the order-disorder transition
[19]. This scenario may naturally explain the logarithmic time dependence, as the structural rearrangements within the core consist of many correlated steps where the chains are forced to move consecutively in a hierarchical manner. However, as this problem constitutes a very complex many-chain problem, further quantitative evaluations appear difficult without any detailed microscopic model.

In summary, we have presented experimental data for three well-defined model systems for diblock copolymer micelles. It has been shown that the exchange kinetics follows a logarithmic time dependence. We suggest that this logarithmic law may be a general feature for sufficiently confined polymeric micelles. The behavior may result from strongly coupled chain dynamics induced by geometrical constraints in the core.

The authors would like to thank ILL for providing beam time on the D-11 instrument, and Dr. Elena Dormidontova and Dr. Michael Monkenbusch for fruitful discussions.

*Electronic address: r.lund@fz-juelich.de

[1] A. Halperin and S. Alexander, Macromolecules 22, 2403 (1989).

[2] E. Dormidontova, Macromolecules 32, 7630 (1999).

[3] K. Prochazka, B. Bednar, E. Mukhtar, P. Svoboda, J. Trnena, and M. Almgren, J. Phys. Chem. 95, 4563 (1991).

[4] Y. Wang, C. Kausch, M. Chun, R. Quirk, and W. Mattice, Macromolecules 28, 904 (1995).

[5] R. Underhill, J. Ding, V. Birss, and G. Liu, Macromolecules 30, 8298 (1997).

[6] G. Waton, B. Michels, and R. Zana, Macromolecules 34, 907 (2001).

[7] T. Haliloglu, I. Bahar, B. Erman, and W. Mattice, Macromolecules 29, 4764 (1996).

[8] V. Karpov and M. Grimsditch, Phys. Rev. B 48, 6941 (1993).

[9] B. Persson, Phys. Rev. B 51, 13568 (1995).

[10] M. Skorobogatiy, H. Guo, and M. Zuckermann, J. Chem. Phys. 109, 2528 (1998).

[11] E. Brauns, M. Madaras, R. Coleman, C. Murphy, and M. Berg, Phys. Rev. Lett. 88, 158101 (2002).

[12] L. Willner, A. Poppe, J. Allgaier, M. Monkenbusch, and D. Richter, Europhys. Lett. 55, 667 (2001).

[13] R. Lund, L. Willner, J. Stellbrink, A. Radulescu, and D. Richter, Macromolecules 37, 9984 (2004).

[14] R. Lund, Ph.D. dissertation, University of Munster, 2004.

[15] R. Palmer, D. Stein, E. Abrahams, and P. Anderson, Phys. Rev. Lett. 53, 958 (1984).

[16] J. Brey and A. Prados, Phys. Rev. E 63, 021108 (2001).

[17] N. Fatkullin, R. Kimmich, E. Fischer, C. Mattea, U. Beginn, and M. Kroutieva, New J. Phys. 6, 46 (2004).

[18] C. Chen and E. Dormidontova (unpublished).

[19] K. Cavicchi and T. Lodge, Macromolecules 36, 7158 (2003). 